\title{
The setup and application of the multi-scale in-situ test system for fatigue damage analysis
}

\author{
Yi Shi ${ }^{1, *}$, Xiaoguang $\mathrm{Yang}^{1}$, Guolei $\mathrm{Miao}^{2}$ and Duoqi $\mathrm{Shi}^{1}$ \\ ${ }^{1}$ Beihang University, School of Energy and Power Engineering, 100191 Beijing, China \\ ${ }^{2}$ Chengdu Holy Industry\& Commerce Corp.LTD, 611936 Sichuan Province, China
}

\begin{abstract}
This essays aims at introducing the setup for the multi-scale in-situ test system which is applied for the fatigue crack initiation test. The setup of the experiment system is first introduced, including the image capturing system, optical path system, image acquisition and storage system and the three-axis mobile platform. Then the preparation of micro speckle and the corresponding technique for spatial adjustment are improved to realize the DIC measurement in micro scale. Finally three experiments from macro-meso scale to macro scale: fatigue initiation test, the observation of micro crack and the fatigue crack growth rate in macro scale were conducted to verify the application of the system. The test result can indicate the location of crack initiation, the crack growth rate and the evolution of displacement/strain field, etc.
\end{abstract}

\section{Introduction}

Fatigue is the main cause of failure of engineering structures and components. The fatigue damage of metal is composed of multiple process which is generally divided into the following stages: 1) the accumulated damage caused by the change in microstructure; 2) the nucleation of micro crack; 3) the crack growth of micro crack and formation of main crack; 4) the crack growth; 5) fracture ${ }^{[1]}$. Therefore fatigue failure is a multi-stage continuous process. The fatigue life is generally analyzed via the post-test fractography after the fatigue tests. However, this can only analyze the existing results instead of revealing the middle process. Actually understanding the intermediate process of fatigue damage will be helpful for analyzing the underlying failure mechanism and identifying key factors.

By now many researchers have applied different methods to study various stages of fatigue damage process. For example, the surface replica is used for the study of small crack growth ${ }^{[2-4]}$; the compliace method and potential drop method are applied to monitor the length of long crack $^{[5-7]}$; the in-situ SEM methods are used to investigate the initation of crack ${ }^{[8-9]}$. However, all these methods have thier limitations. More specifically, the process of surface replica is complicated and inefficient. The specimen should be notched to ensure the crack can be captured inthe view field of SEM. Additionally, some methods can only acquire the length of long cracks. Therefore, a new experimental setup which can realize the in-situ multi-measurement based on the hydraulic fatigue testing machine is proposed.

\section{Experiment system}

The experiment system is composed of four subsystems, including 1) image capturing system, 2) optical path system, 3) image acquisition and storage system and 4) three-axis mobile platform (Fig. 1). This system cooperates with the axial servo-hydraulic testing machine instead of tensile stage to realize the in-situ observation and measurement of related parameters in the loading process. It is widely accepted that the performance of cyclic loading from hydraulic testing machine is better than the tensile stage.

\subsection{Image capturing system}

The image capturing system includes optical lens and camera components. To ensure the resolution for the meso and micro scale, the selection of CCD camera and sensor should be careful. In this system, the German manufactured high resolution $\mathrm{CCD}$ camera named AVT-GT2450 is applied, with $2 / 3$ inch sensor and $2448 \times 2050$ pixels resolution. The optical lens can achieve high resolution lens with 1.16-66.63 times zoom, with $0.17-9.49 \mathrm{~mm}$ (diagonal length) working view field, which is situable for the meso scale. For the measurement in the macro scale, the present lens can be replaced with bilateral telecentric lens and combined with CCD camera ${ }^{[10]}$ 


\subsection{Optical Path System}

The coaxial optical path system is applied in this system. Compared with the traditional ring LED light source, the coaxial optical path system could offer more uniform light which can improve the accuracy and reproducibility of machine vision. Therefore it is more convenient for the observation of surface information such as foreign object, crack and scratches. Meanwhile, to satisfy the light intensity requirements as well as the stable light source for the experiment, the high-intensity LED cold light source with fiber is applied. This light source can not only produce less heat under the working condition, but also provide long-lasting light.

\subsection{Image Acquisition and Storage System}

The Stream Pix software is selected for the image storage and acquisition. This software has capacity of high-frequency image acquisition and continuous or fixed-interval image storage.

\subsection{Three-axis mobile platform}

The main functions of the three-axis mobile platform includes 1) support the whole image capture system; 2) to reduce the vibration from other objects; 3 ) to adjust the focal length of lens. This platform is made up of three parts: 1) vibration isolation base platform; 2) three-axis coarse adjustment mobile platform; 3 ) threeaxis fine adjustment mobile platform. More specifically, the main function of the three-axis adjustment mobile platform is to adjust the focal length of lens. For the observation and measurement in meso scale, the lens should be set at large zoom. However, with the increase in zoom rate, the depth of filed will decreases. If the resolution of fine-adjustment is low, it will be difficult to find the appropriate focal length for the lens. In addition, images are likely to be influenced significantly by small vibration. Therefore, it is necessary to apply good vibration isolation system.

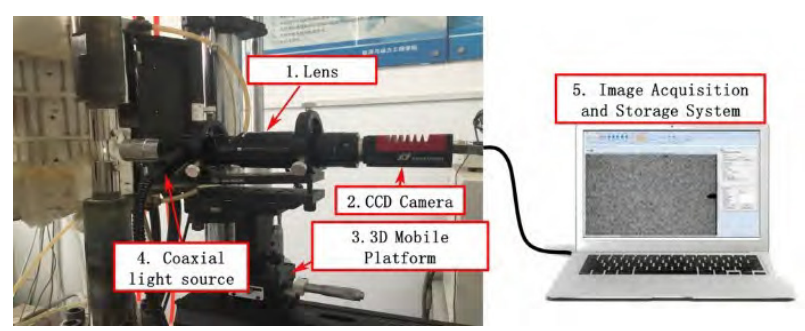

Fig. 1. The setup of the in-situ system

\section{Digital image correlation (DIC) method}

\subsection{DIC basic theory}

Digital image correlation method, as known as the digital speckle correlation method is different from the traditional contacting measurement such as extensometer, which is based on the image processing and numerical calculation and is a kind of non-contact whole-field deformation optical measurement method ${ }^{[11]}$. The basic principle is to calculate the corresponding displacement filed and strain field by analyzing (speckle or metal surface texture) deformation and displacement of the information carrier in the deformed images and unreformed images, shown in Fig. 2. The process of DIC method is divided into two parts: 1) matching the acquired deformed images and initial images; 2) calculating the displacement field in the region of interest and calculate the strain field DIC through the differential displacement field. In the DIC algorithm, the images are matched by the grey value in the subset. In other words, the correlation function is used to analyze the pre-deformation and post-deformation values and match tem based on the maximum correlation value in the process of DIC image. If the grey value of the deformed carrier is changed during the test, it will cause the DIC algorithm fail to further calculate the displacement field or deformation field. Therefore the quality of speckle is a key problem in the realization of DIC technique. It is required that the speckles should have the random grey value distribution. If the gradation is concentrated in a range, it will make it difficult to match in the operation.
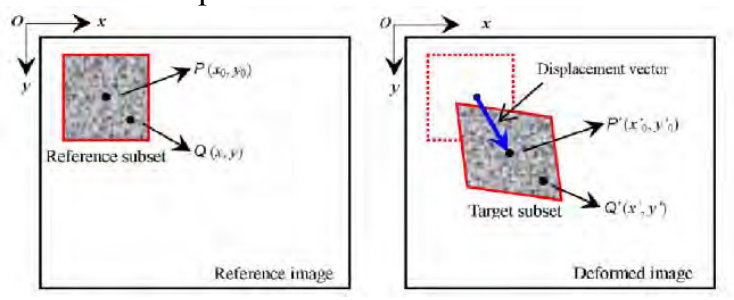

Fig. 2. DIC algorithm schematic

\subsection{Preparation for the micro speckle}

The speckle preparation is the key to achieve accurate measurement of DIC and the production of speckles for small scales it is important to the crack initiation test. In this paper, the micro speckles were produced by spraying method. The black graphite powder with a particle diameter of $10 \mu \mathrm{m}$ was used as the "paint". The graphite powder was sprayed with an airbrush and attached to the surface of specimen by means of electrostatic adsorption. This speckle preparation method is convenient, however to obtain high quality speckle the preparation parameter should be adjusted. For example the pressure of the airbrush, the spray angle and distance. To ensure the resolution of DIC test, the quality of the prepared speckles need to be evaluated. The global parameter estimation method based on average gray gradient of speckle images proposed by Pan Bing ${ }^{[12-13]}$ was applied. The average gray gradient of this speckle is 15.46 , and the speckle image and grey value distribution in shown in Fig. 3. 


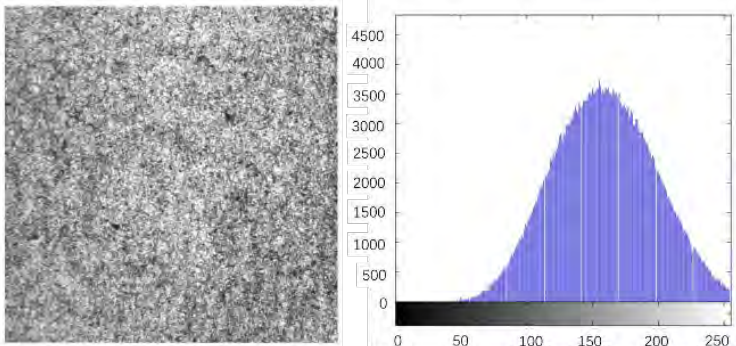

Fig. 3. The image of speckle made by graphit powder and its gray level distribution histogram

\subsection{Spatial adjustment technique for the platform}

Besides the quality of deformed carrier, the adjustment of the 3D mobile platform also makes difference to the test results. For the 2D-DIC test, since the out-of-plane displacement has a detrimental effect on the result, it must be ensured that the optical axis of the camera lens is in a spatial vertical state with the sample surface, shown in Fig. 4. The detailed steps are given below: 1) To ensure that the optical axis of the lens and the loading axis of the fatigue testing machine are in a plane perpendicular state, the pitch of the 3D mobile platform must be adjusted and the lens should be adjusted to a horizontal level with a level instrument; 2) to ensure the spatial vertical state of the optical axis and test specimen surface, the specimen should be rotated for adjustment. In addition to the empirical operation, a little load can be given (at the premise of not influencing the test result) to conduct and compare the displacement field distribution to the theoretical one. If the results is consistent, the lens are well perpendicular to the sample surface.

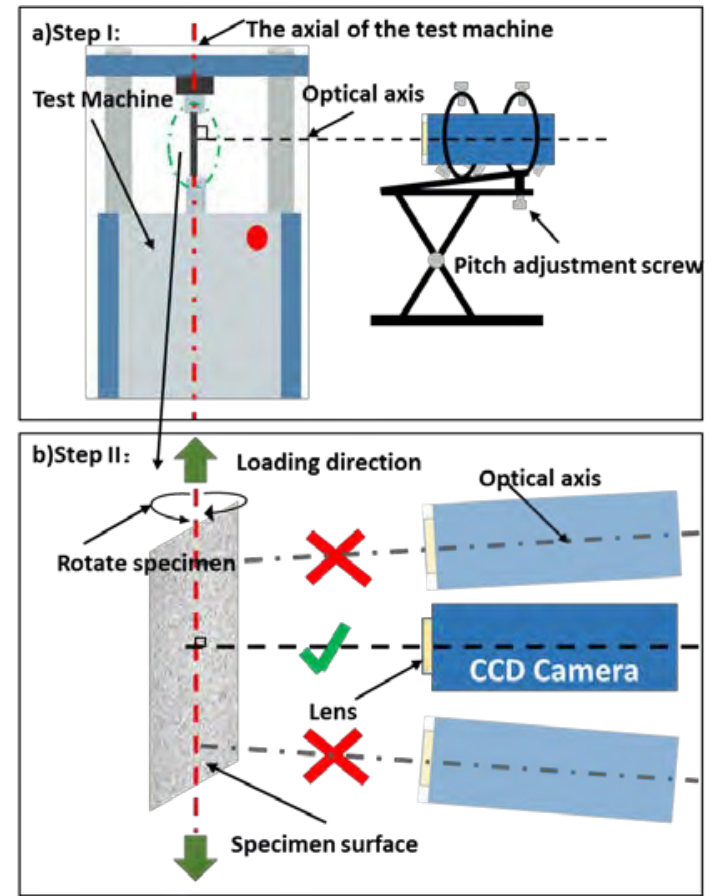

Fig. 4. The schematic of test equipment setting requirements and steps

\section{Application for the Testing in Macro- meso Scale}

\subsection{The observation of crack initiation test}

The purpose of this test is to realize the observation of crack initiation at room temperature combined with DIC method. Generally, the position of the crack initiation is difficult to locate at early stage by naked eyes because these cracks are too small and stochastic as well. However, with the help of contour calculated by DIC method, it can indicate the position due to the discontinuity at the surface.

\subsubsection{Test specimen and procedure}

GH536 is a kind of Chinese made nickel based superalloys which is applied in aero-engine flame tube of combustion chamber due to its excellent mechanical performance at elevated temperature. Its average grain size is approximately 50 micro meter. The grain size should be taken into consideration when prepare the speckle and design the gauge section. To identify the deformation between grains, there should be certain amount of speckles in one grain. In addition, the size of gauge section is related to the magnification rate. Lower magnification rate results in large pixel point thus there will be less speckles in one grain. In this test, the gauge section is $2 \mathrm{~mm} \times 2 \mathrm{~mm}$ (Fig. 5) and the magnification rate of lens is 7 times.

Before the preparation for the micro speckle, the specimen was polished by abrasive paper in the order from 800-3000 to the mirror surface. The purpose of this procedure is to eliminate the scratches which will exert negative effect on the strain field measurement. Finally, the U-STAR GP-300 airbrush with $0.5 \mathrm{~mm}$ caliber was applied to manufacture the speckles.

\subsubsection{Test Condition}

The triangular wave with maximum load $3.0 \mathrm{kN}$ was applied in the test. The loading ratio was set at 0.1 with frequency $3 \mathrm{~Hz}$. The test was conducted at room temperature and controlled by loading. The test was interrupted for image capturing. In other words, a complete cycle of image information was captured at regular intervals (Fig. 6).In the capture progress, the loading rate was $0.1 \mathrm{kN} / \mathrm{s}$.

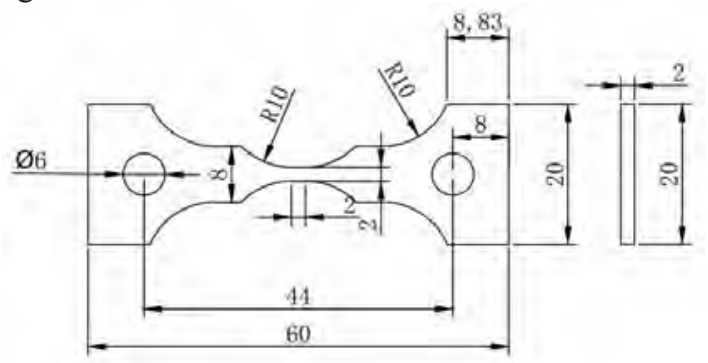

Fig. 5. The schematic of the test specimen 


\subsubsection{Result and analysis}

When DIC analysis software is used to analyze the deformation field in the process of fatigue damage evolution, the types of strain fields obtained are different depending on the selection of reference pictures and deformation pictures. If the image of the specimen at the minimum loading in the first cycle is chosen as the reference image and other images at the peak loading is treated as deformed image, the evolution of the total strain field at the specimen surface in the fatigue progress can be obtained and analyzed (Fig. 7). On the other hand, if images taken at the minimum loading in each cycle were chosen as deformed image, the residual strain filed could be obtained (Fig. 8).

1) The analysis of total strain field

The calculation of total strain field is useful for locate the initiation of micro cracks. The physical size of micro crack is small and it is usually hard to find until it reaches hundred micro meter. Although the sprayed speckles on the surface seem to add difficulty in observation, the application of DIC method indicates the micro cracks. Since the DIC algorithm is based on the correlation between the deformed image and reference image. If the subset fails to find the corresponding subset in the deformed image due to the discontinuity on the surface caused by the micro cracks, there will be no calculation for this subset. In other words, this area will be 'faded' in the contour images. In addition, the value of strain can be calculated based on the DIC algorithm mentioned before. Therefore, in the process of analysis, the location of potential crack can be identified by the color of contour. The results of this test is shown in Fig. 9.In early stage, for example Fig. 9(b)-(c), six points were chosen from higher strain area. Meanwhile the strain value of each point was extracted. The diagram of evolution of total maximum strain was also plotted Fig. 10. Among the six points, before 40000 cycles point $B$ took the first place. However, point $\mathrm{C}$ which was the smallest one initially exceeded point B after 40000 cycles. A significant increase can be observed for all points at approximately 4000 cycles except point F. Point $\mathrm{F}$ first stabilized at 0.02 before 4000 cycles and then grew less significantly than other points to 0.022 . The contour at 46135 cycles is shown in Fig. 11. Micro cracks can be observed by the 'missing color' area. Meanwhile, the strain value of circled points is higher than other area, which further indicate these points are likely to be the initiation places. For point $\mathrm{F}$ which appeared higher strain value than point A, C, D, E at the beginning, no sign of initiation can be found,Fig. 9(g)(h). On one hand, the strain value is obviously lower compared with other five points. On the other hand, there is no discontinuity in the contour in the point $\mathrm{F}$ area. Therefore, the application of DIC method is effective for identifying the initiation places. The value of strain also makes difference. From this test, some area like point $\mathrm{F}$ exhibits higher strain rate at first but does not initiate earlier than others. In this view filed, there were several micro cracks at the surfaces. If the advanced microscope is applied for this specimen, only one or even none cracks could fall into this view field and thus losing important information of the evolution. The specimen fractured under another 1919 cycles after 46135 cycles. This also means there are only little time left for obersevation when the micro cracks can be found by naked eyes.

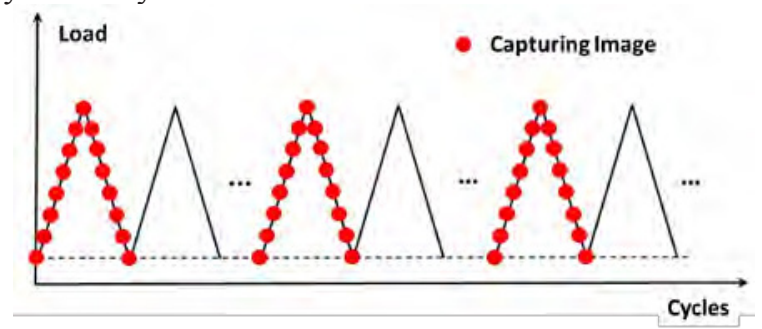

Fig. 6. Image information acqusition spectrum during loading

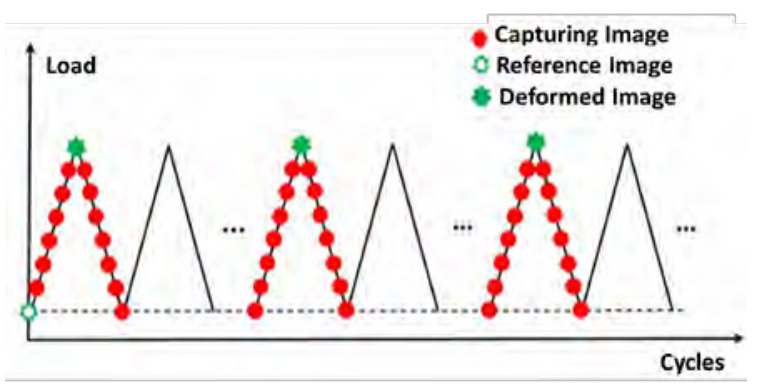

Fig. 7. The schematic of calculation for the maximum total strain by DIC anaylsis

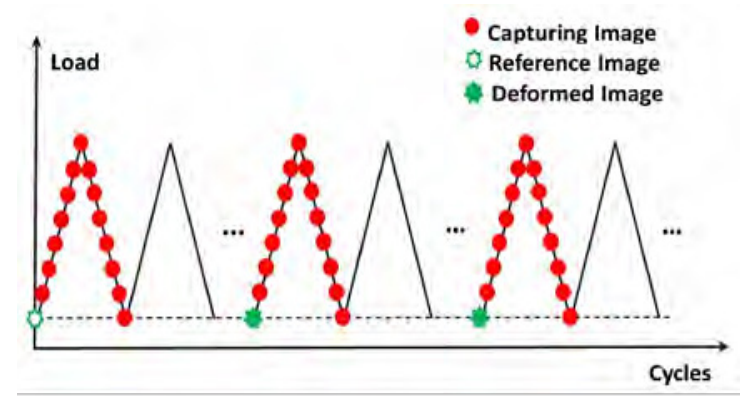

Fig. 8. The schematic of calculation for the residual strain by DIC analysis 

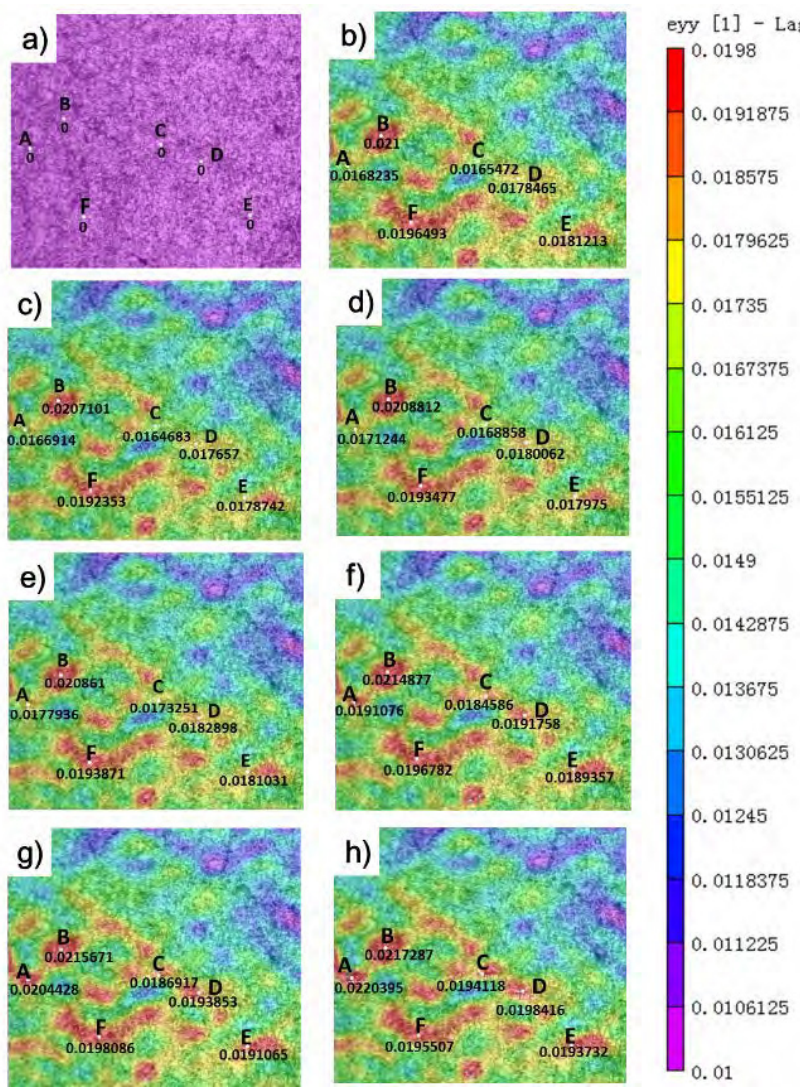

Fig. 9. The contour of the maximum total strain evolution in different cycles a) unloading; b) 3002 cycles; c) 13004 cycles; d) 18005 cycles; e) 23006 cycles; f) 29727 cycles; g) 30727 cycles

2) The analysis of residual strain field

The residual strain can be calculated by DIC as mentioned before. The correlation method is a kind of subtraction. In other words it compares the difference between unloading at different cycles and thus indicates the residual strain field. By extracting the value from images, the curve of the accumulated residual strain is shown in Fig. 12 Similar to the curve of total strain evolution, an obvious increase can be observed near 40000 cycles. The accumulated strain first stabilized at $1.6 \%$ and then increased slightly to $1.7 \%$. However, after 40000 cycles, it soared to $2.6 \%$ with another 5000 cycles. The curve also reflect that initiation stage consumes most fatigue life.

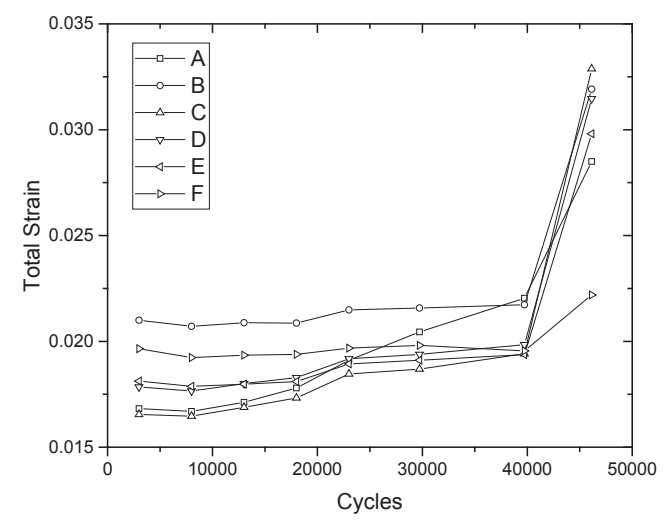

Fig. 10. The total maxmium strain evolution of six points

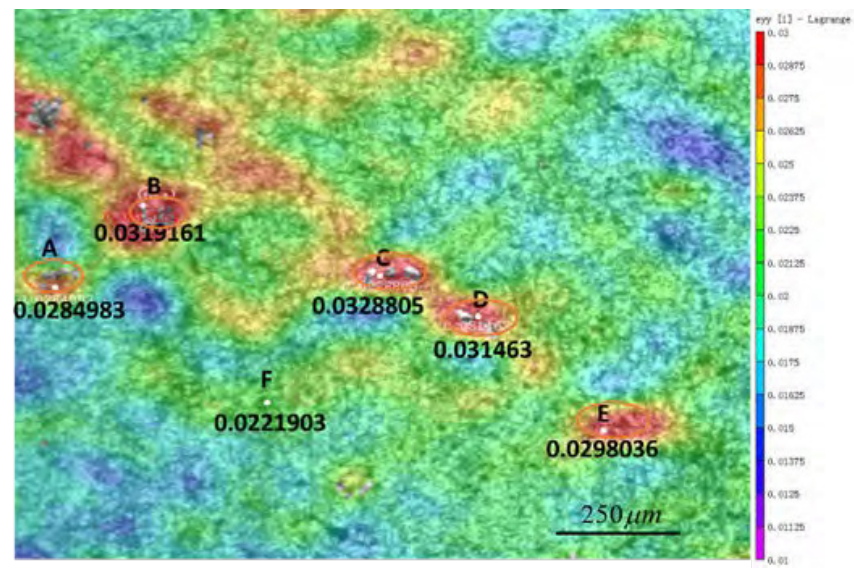

Fig. 11. The contour of total strain at 46135 cycles

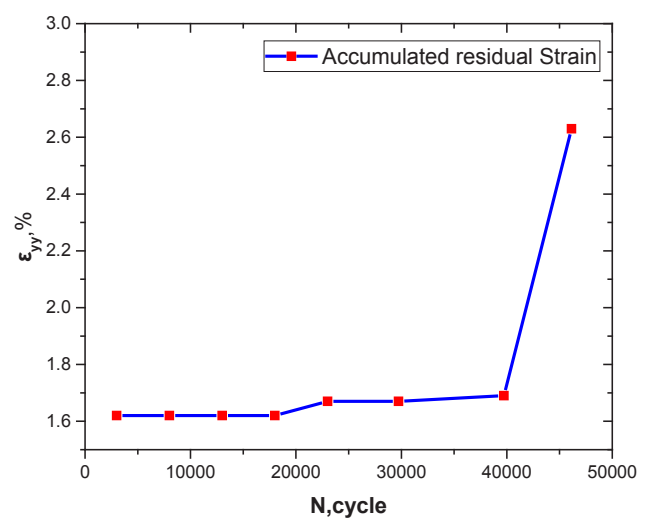

Fig. 12. The cruve of the accumulated residual strain

\subsection{The observation of small crack growth}

As is mentioned before, surface replica and some in-situ observation instruments are applied in investigation of small crack growth. For the setup of this in-situ system, it is also effective for this purpose.

\subsubsection{Test specimen and procedure}

The material of the specimen is a Chinese made nickel based powder metallurgy superalloys named FGH96. The geometry of the specimen is shown in Fig. 13, and the notch is done by EDM. In order to characterize the grain feature, the surface of the specimen was corroded by the etchant solution: copper chloride $5 \mathrm{~g}+$ ethyl alcohol $100 \mathrm{ml}+$ hydrochloric acid $100 \mathrm{ml}$. The grain character and twin grains can be observed from our in-situ setup Fig. 14. The test condition is similar to the previous one but the maximum loading in this case is $4 \mathrm{kN}$. 


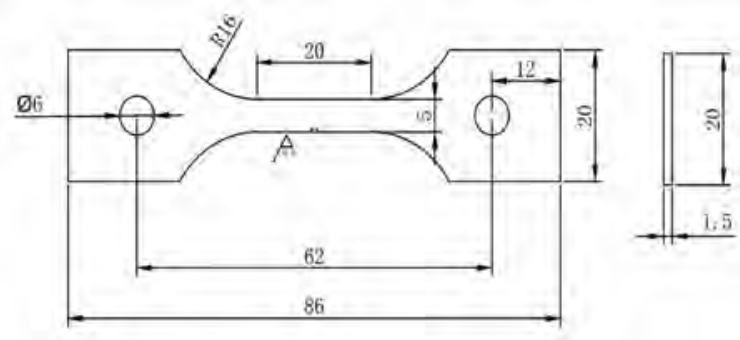

Fig. 13. The schematic of the test specimen

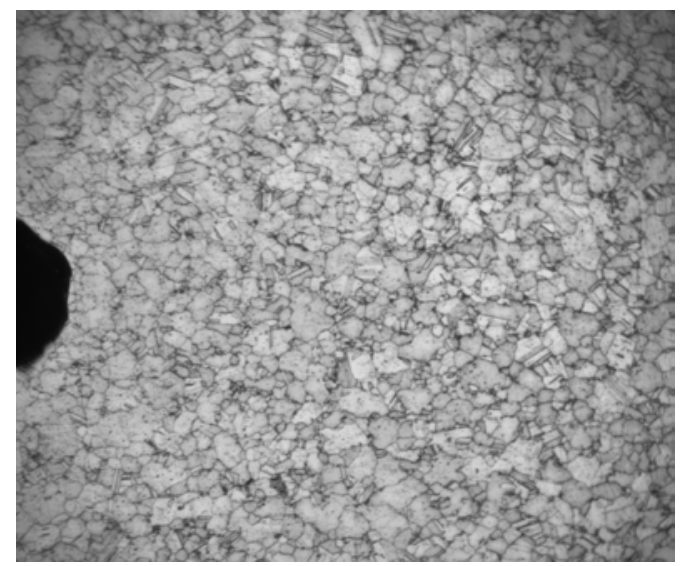

Fig. 14. The image of the corroded specimen captured by the setup

\subsubsection{Result and analysis}

The crack imitation mode and path can be clearly captured. In Fig. 15, no crack can be observed before the 5002nd cycle. Later, at the 14341 st cycle, a micro crack can be detected in the grain near the bottom of the notch. The initiation mode is identified as inner-grain initiation mode. In the process of crack growth, intergranular and transgranular changes alternately (Fig. 16). The crack path first grew in the 45 degree from the loading direction, and the direction changes continuously instead of being perpendicular. This is mainly due to the unevenly distributed grain size and orientation. The $45^{\circ}$ defelection also indicates the inital failure was casued by the maximum shear stress.

According to the definition of initiation, microstructure small crack, physically small crack and long crack, the crack growth can be divided into four stages. The physically small crack is usually defined as the plastic area is larger than the average microstructure size and is smaller than $1 \mathrm{~mm}$. For FGH96, the average grain size is $23.8 \mu \mathrm{m}$ measured by EBSD in our previous work ${ }^{[14]}$. Therefore, the minimum size of the physically small crack should be 3-5 grains size. The percentage of each stage accounting for is shown in Fig. 17. The initiation stage accounts almost half part of the total life. Meanwhile, the small crack stage contributed approximately $50 \%$. Only $4 \%$ life belongs to the long crack growth. Therefore, the stage of small crack cannot be neglected. Future efforts will be put on the study of physically small crack growth. The relationship between cycle number and crack length is given in Fig. 18. The crack growth rate at the transition point between the PSC and MSC increased significantly while the rate in the PSC decreased to 27500 cycle and then increased evidently until the LC.
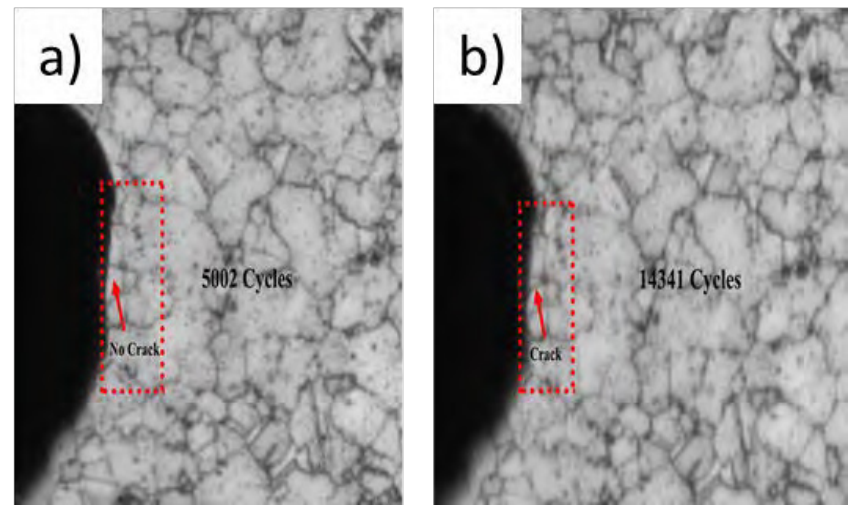

Fig. 15 Initiation in one grain a) the 5002th cycle; b) the 14341st cycle

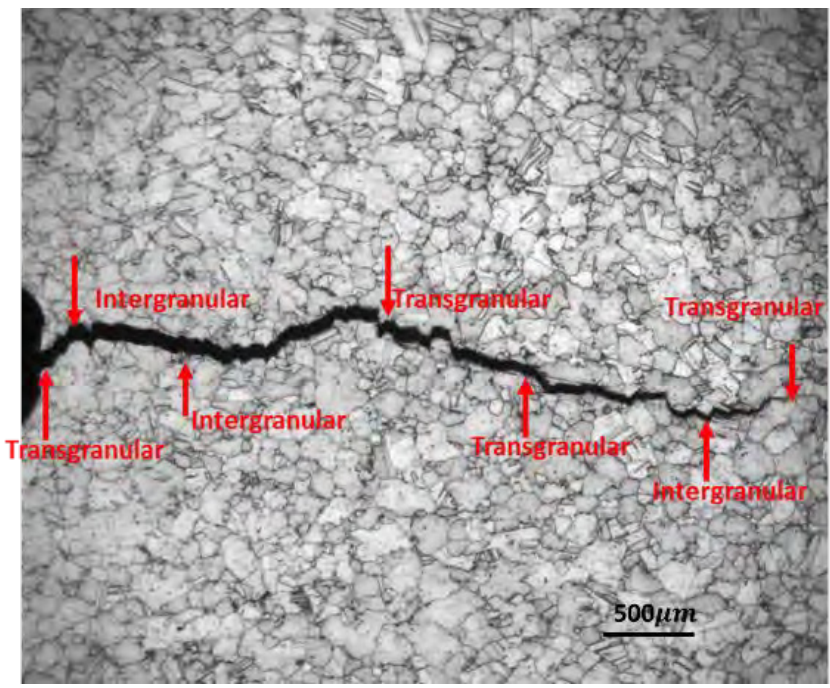

Fig. 16. The crack growth path (the 32869th cycle)

\section{Application for the Testing in macro Scale}

This multi-scale in-situ test system also has the capability of monitoring the long crack growth in macro scale. The deformation of the surface is also indicated by the DIC method.

\subsection{Test specimen and procedure}

The single edge notch tension (SENT) specimen was applied in this test, shown in Fig. 19. The specimen was designed based on the E647 standard ${ }^{[15]}$. Compared with the traditional compact tension specimen, the SENT has a wider area for observation. The surface of the SENT was polished by the abrasive paper to the mirror state. To enhance the image contrast, white paint was used as the background and black paint was chosen as the deformation carrier. The maximum loading was $5 \mathrm{kN}$, with the R- 0.1 at the frequency $2 \mathrm{~Hz}$. The capture method is similar to the previous mentioned tests. 


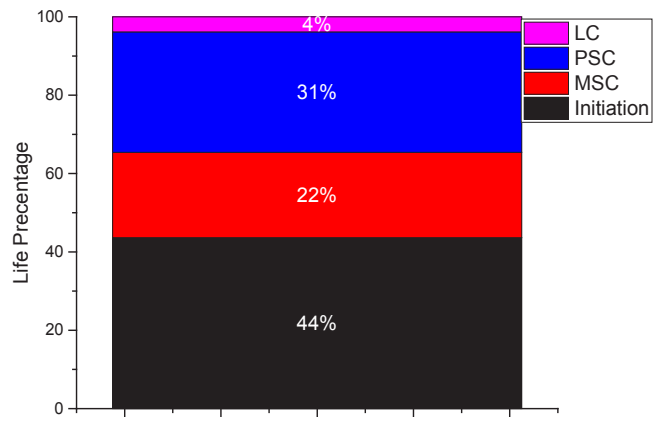

Fig. 17. The percentage of each stage of the taotal life

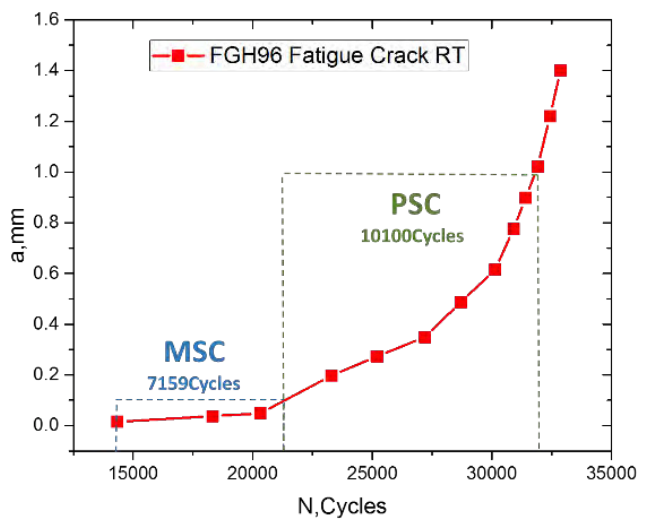

Fig. 18. The relationship between the crack legnth and cycles
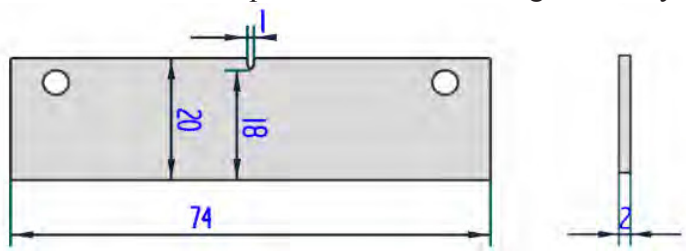

Fig. 19. The geometry of the SENT specimen

\subsection{Result and analysis}

The fatigue crack length can be obtained by the image captured by this system and the stress intensity can be calculated by the formula provided by the E647. The fatigue crack growth starts from $10^{-5} \mathrm{~mm} /$ cycle to $10^{-3} \mathrm{~mm} /$ cycle and the stress intensity falls between 30 to $65 \mathrm{MPa} \cdot \mathrm{m}^{1 / 2}$. In the medium and higher level of stress intensity factor, the data points is linear in the logarithm scale, shown in Fig. 20 which is consistent with the Paris law.

The strain can be calculated by DIC method which is similar to the method described beforehand. The evolution of strain field is shown in Fig. 21. The shape of the plastic zone at the crack tip is consistent with the plastic shape of the von Mises principle in the plane stress condition. Under the cylic loading, the strain at the crack tip increased significantly. In addition, the contour of the area along the crack seems faded due to the discontinuity which was discussed before. By extracting the maximum strain, the relationship between the strain and stress intensity factor is linear, shown in figure Fig. 22.

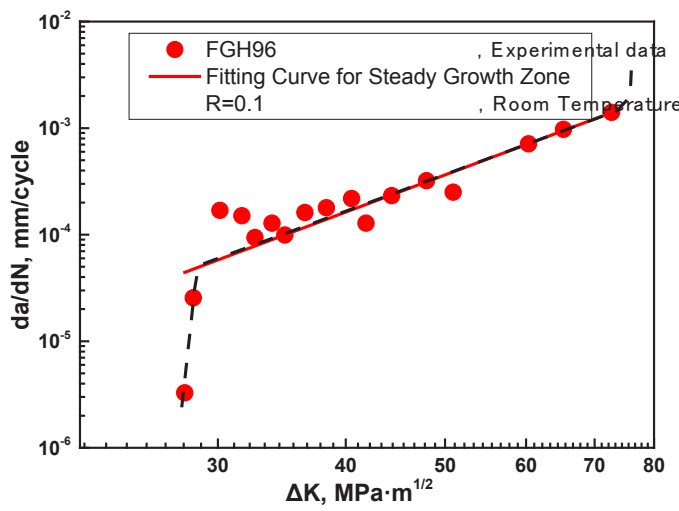

Fig. 20. The fatigue crack growth rate of FGH96

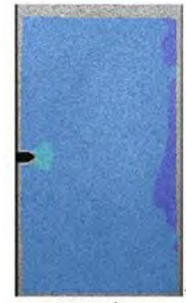

19006 cycles

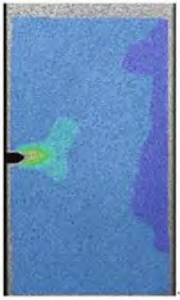

32515 cycles

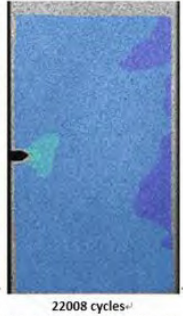

22008 cycles

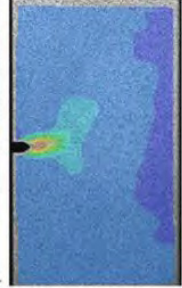

35519 cycles.

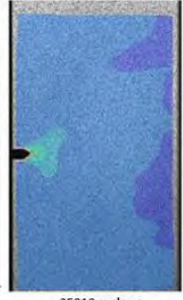

25010 cycles

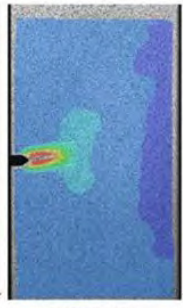

30720 cycles

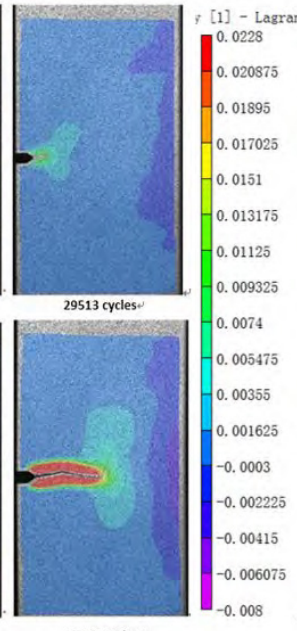

39505 cycles
Fig. 21. The evolution of maximum strain of SENT specimen

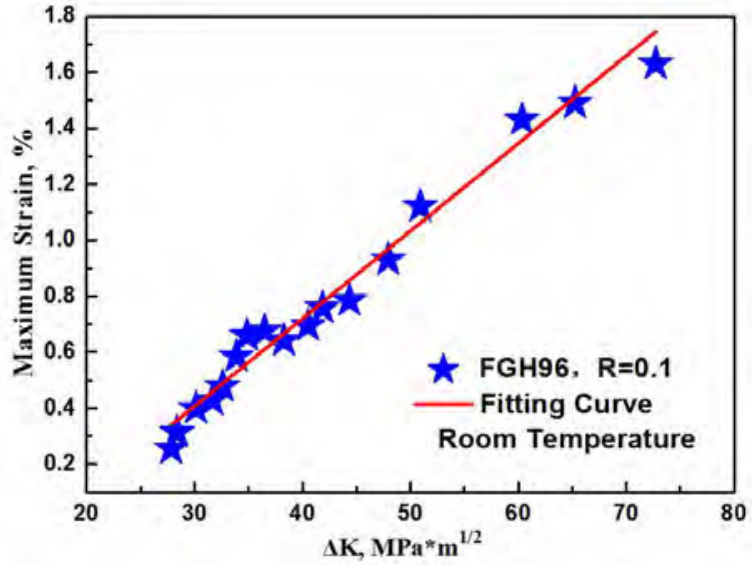

Fig. 22. The relationship between the maximum strain and stress intensity factor

The authors would like to thank the sponsorship by National Program on Key Basic Research Project (973 Program) (No. 2015CB057401). 


\section{References}

1. J.Schijve, Fatigue of Structures and Materials (2001).

2. M.Goto,D.Knowles, Eng. Fract. Mech. 60(1), 1-18 (1998).

3. J.Newman,S.Willard,S.Smith,R.Piascik, Eng. Fract. Mech., 76(7), 898-910 (2009).

4. K.Zhang,X.Wu,C.Davies, Int. J. Fatigue., 104 (2017).

5. R.Bao, H.Yang, J.Zhang, L.Peng, B.Fei, Int. J. Fatigue., 47(1), 189-195 (2013)

6. X.He,Y.Zhang,H.Shi,J.Gu,C.Li,K.Kai, Mater. Sci. Eng. A, 618 153-160 (2014)

7. S.Na,D.Yoon,J.Kim,H.Kim,D.Kim, Int. J. Fatigue., 101 27-35 (2017)

8. H.Pang,P. Reed. Mater. Sci. Eng. A, 448(1) 67-79 (2007)

9. R.Jiang,F.Pierron,S.Octaviani,P.Reed, Mater.Sci.En g. A, (2017).

10. B.Pan, Acta Opt. Sin., 33(4) (2013)

11. H.Gao,H.Liu,Z.Qi,H.Zheng, Ordnance Mater. Sci. Eng. (2016).

12. B.Pan,Z.Lu,H.Xie.Optics \& Lasers in Eng. 48(4) 469-477 (2010)

13. B.Pan,D.Wu,Y.Xia, J. Exp. Mech. 25(2) 120-129 (2010)

14. G.Miao,X.Yang,D.Shi, Mater. Sci. Eng. A, 668 6672 (2016)

15. ASTM International. Standard test method for measurement of fatigue crack growth rates E647-15 (2015) 